\title{
REGULAR PSEUDO-HYPEROVALS AND REGULAR PSEUDO-OVALS IN EVEN CHARACTERISTIC
}

\author{
J. A. THAS
}

\begin{abstract}
S. Rottey and G. Van de Voorde characterized regular pseudo-ovals of $\mathbf{P G}(3 n-1, q), q=2^{h}, h>1$ and $n$ prime. Here an alternative proof is given and slightly stronger results are obtained.
\end{abstract}

\section{INTRODUCTION}

Pseudo-ovals and pseudo-hyperovals were introduced in [10]; see also [12]. These objects play a key role in the theory of translation generalized quadrangles $[6,12]$. Pseudo-hyperovals only exist in even characteristic. A characterization of regular pseudo-ovals in odd characteristic was given in [2]; see also [12]. In [8] a characterization of regular pseudo-ovals and regular pseudo-hyperovals in $\mathbf{P G}(3 n-1, q)$, $q$ even, $q \neq 2$ and $n$ prime, is obtained. Here a shorter proof is given and slightly stronger results are obtained.

\section{OVALS AND HYPEROVALS}

A $k$-arc in $\mathbf{P G}(2, q)$ is a set of $k$ points, $k \geq 3$, no three of which are collinear. Any non-singular conic of $\mathbf{P G}(2, q)$ is a $(q+1)$-arc. If $\mathcal{K}$ is any $k$-arc of $\mathbf{P G}(2, q)$, then $k \leq q+2$. For $q$ odd $k \leq q+1$ and for $q$ even a $(q+1)$-arc extends to a $(q+2)$-arc; see [3]. A $(q+1)$-arc is an oval; a $(q+2)$-arc, $q$ even, is a complete oval or hyperoval.

A famous theorem of B. Segre [9] tells us that for $q$ odd every oval of $\mathbf{P G}(2, q)$ is a non-singular conic. For $q$ even, there are many ovals that are not conics [3]; also, there are many hyperovals that do not contain a conic [3].

\section{GENERALIZED OVALS AND HYPEROVALS}

Arcs, ovals and hyperovals can be generalized by replacing their points with $m$ dimensional subspaces to obtain generalized $k$-arcs, generalized ovals and generalized hyperovals. These have strong connections to generalized quadrangles, projective planes, circle geometries, flocks and other structures. See [6, 12, 10, 11, 2, 7]. Below, some basic definitions and results are formulated; for an extensive study, many applications and open problems, see [12].

A generalized $k$-arc of $\mathbf{P G}(3 n-1, q), n \geq 1$, is a set of $k(n-1)$-dimensional subspaces of $\mathbf{P G}(3 n-1, q)$ every three of which generate $\mathbf{P G}(3 n-1, q)$. If $q$ is odd then $k \leq q^{n}+1$, if $q$ is even then $k \leq q^{n}+2$. Every generalized $\left(q^{n}+1\right)$-arc of $\mathbf{P G}(3 n-1, q), q$ even, can be extended to a generalized $\left(q^{n}+2\right)$-arc. 
If $\mathcal{O}$ is a generalized $\left(q^{n}+1\right)$-arc in $\mathbf{P G}(3 n-1, q)$, then it is a pseudo-oval or generalized oval or [n-1]-oval of $\mathbf{P G}(3 n-1, q)$. For $n=1$, a [0]-oval is just an oval of $\mathbf{P G}(2, q)$. If $\mathcal{O}$ is a generalized $\left(q^{n}+2\right)$-arc in $\mathbf{P G}(3 n-1, q), q$ even, then it is a pseudo-hyperoval or generalized hyperoval or $[n-1]$-hyperoval of $\mathbf{P G}(3 n-1, q)$. For $n=1$, a [0]-hyperoval is just a hyperoval of $\mathbf{P G}(2, q)$.

If $\mathcal{O}=\left\{\pi_{0}, \pi_{1}, \cdots, \pi_{q^{n}}\right\}$ is a pseudo-oval of $\mathbf{P G}(3 n-1, q)$, then $\pi_{i}$ is contained in exactly one $(2 n-1)$-dimensional subspace $\tau_{i}$ of $\mathbf{P G}(3 n-1, q)$ which has no point in common with $\left(\pi_{0} \cup \pi_{1} \cup \cdots \cup \pi_{q^{n}}\right) \backslash \pi_{i}$, with $i=0,1, \cdots, q^{n}$; the space $\tau_{i}$ is the tangent space of $\mathcal{O}$ at $\pi_{i}$. For $q$ even the $q^{n}+1$ tangent spaces of $\mathcal{O}$ contain a common $(n-1)$-dimensional space $\pi_{q^{n}+1}$, the nucleus of $\mathcal{O}$; also, $\mathcal{O} \cup\left\{\pi_{q^{n}+1}\right\}$ is a pseudo-hyperoval of $\mathbf{P G}(3 n-1, q)$. For $q$ odd, the tangent spaces of a pseudo-oval $\mathcal{O}$ are the elements of a pseudo-oval $\mathcal{O}^{*}$ in the dual space of $\mathbf{P G}(3 n-1, q)$.

\section{REgULAR PSEUDO-OVALS AND PSEUDO-HYPEROVALS}

In the extension $\mathbf{P G}\left(3 n-1, q^{n}\right)$ of $\mathbf{P G}(3 n-1, q)$, consider $n$ planes $\xi_{i}, i=1,2, \cdots, n$, that are conjugate in the extension $\mathbb{F}_{q^{n}}$ of $\mathbb{F}_{q}$ and which span $\mathbf{P G}\left(3 n-1, q^{n}\right)$. This means that they form an orbit of the Galois group corresponding to this extension and span PG $\left(3 n-1, q^{n}\right)$.

In $\xi_{1}$ consider an oval $\mathcal{O}_{1}=\left\{x_{0}^{(1)}, x_{1}^{(1)}, \cdots, x_{q^{n}}^{(1)}\right\}$. Further, let $x_{i}^{(1)}, x_{i}^{(2)}, \cdots, x_{i}^{(n)}$, with $i=0,1, \cdots, q^{n}$, be conjugate in $\mathbb{F}_{q^{n}}$ over $\mathbb{F}_{q}$. The points $x_{i}^{(1)}, x_{i}^{(2)}, \cdots, x_{i}^{(n)}$ define an $(n-1)$-dimensional subspace $\pi_{i}$ over $\mathbb{F}_{q}$ for $i=0,1, \cdots, q^{n}$. Then, $\mathcal{O}=\left\{\pi_{0}, \pi_{1}, \cdots, \pi_{q^{n}}\right\}$ is a generalized oval of $\mathbf{P G}(3 n-1, q)$. These objects are the regular or elementary pseudo-ovals. If $\mathcal{O}_{1}$ is replaced by a hyperoval, and so $q$ is even, then the corresponding $\mathcal{O}$ is a regular or elementary pseudo-hyperoval.

All known pseudo-ovals and pseudo-hyperovals are regular.

\section{CHARACTERIZATIONS}

Let $\mathcal{O}=\left\{\pi_{0}, \pi_{1}, \cdots, \pi_{q^{n}}\right\}$ be a pseudo-oval in $\mathbf{P G}(3 n-1, q)$. The tangent space of $\mathcal{O}$ at $\pi_{i}$ will be denoted by $\tau_{i}$, with $i=0,1, \cdots, q^{n}$. Choose $\pi_{i}, i \in\left\{0,1, \cdots, q^{n}\right\}$, and let $\mathbf{P G}(2 n-1, q) \subseteq \mathbf{P G}(3 n-1, q)$ be skew to $\pi_{i}$. Further, let $\tau_{i} \cap \mathbf{P G}(2 n-1, q)=\eta_{i}$ and $\left\langle\pi_{i}, \pi_{j}\right\rangle \cap \mathbf{P G}(2 n-1, q)=\eta_{j}$, with $j \neq i$. Then $\left\{\eta_{0}, \eta_{1}, \cdots, \eta_{q^{n}}\right\}=\Delta_{i}$ is an $(n-1)$-spread of $\mathbf{P G}(2 n-1, q)$.

Now, let $q$ be even and let $\pi$ be the nucleus of $\mathcal{O}$. Let $\mathbf{P G}(2 n-1, q) \subseteq \mathbf{P G}(3 n-1, q)$ be skew to $\pi$. If $\zeta_{j}=\mathbf{P G}(2 n-1, q) \cap\left\langle\pi, \pi_{j}\right\rangle$, then $\left\{\zeta_{0}, \zeta_{1}, \cdots, \zeta_{q^{n}}\right\}=\Delta$ is an $(n-1)$-spread of $\mathbf{P G}(2 n-1, q)$.

Next, let $q$ be odd. Choose $\tau_{i}$, with $i \in\left\{0,1, \cdots, q^{n}\right\}$. If $\tau_{i} \cap \tau_{j}=\delta_{j}$, with $j \neq i$, then $\left\{\delta_{0}, \delta_{1}, \cdots, \delta_{i-1}, \pi_{i}, \delta_{i+1}, \cdots, \delta_{q^{n}}\right\}=\Delta_{i}^{\star}$ is an $(n-1)$-spread of $\tau_{i}$.

Finally, let $q$ be even and let $\mathcal{O}=\left\{\pi_{0}, \pi_{1}, \cdots, \pi_{q^{n}+1}\right\}$ be a pseudo-hyperoval in $\mathbf{P G}(3 n-1, q)$. Choose $\pi_{i}$, with $i \in\left\{0,1, \cdots, q^{n}+1\right\}$, and let $\mathbf{P G}(2 n-1, q) \subseteq$ $\mathbf{P G}(3 n-1, q)$ be skew to $\pi_{i}$. Let $\left\langle\pi_{i}, \pi_{j}\right\rangle \cap \mathbf{P G}(2 n-1, q)=\eta_{j}$, with $j \neq i$. Then $\left\{\eta_{0}, \eta_{1}, \cdots, \eta_{i-1}, \eta_{i+1}, \cdots, \eta_{q^{n}+1}\right\}=\Delta_{i}$ is an $(n-1)$ - spread of $\mathbf{P G}(2 n-1, q)$.

Theorem 5.1 (Casse, Thas and Wild [2]). Consider a pseudo-oval $\mathcal{O}$ with $q$ odd. Then at least one of the $(n-1)$-spreads $\Delta_{0}, \Delta_{1}, \cdots, \Delta_{q^{n}}, \Delta_{0}^{\star}, \Delta_{1}^{\star}, \cdots, \Delta_{q^{n}}^{\star}$ is regular 
if and only if they all are regular if and only if the pseudo-oval $\mathcal{O}$ is regular. In such a case $\mathcal{O}$ is essentially a conic over $\mathbb{F}_{q^{n}}$.

Theorem 5.2 (Rottey and Van de Voorde [8]). Consider a pseudo-oval $\mathcal{O}$ in PG $(3 n-$ $1, q)$ with $q=2^{h}, h>1, n$ prime. Then $\mathcal{O}$ is regular if and only if all $(n-1)$-spreads $\Delta_{0}, \Delta_{1}, \cdots, \Delta_{q^{n}}$ are regular.

\section{Alternative PROOF AND IMPROVEMENTS}

Theorem 6.1. Consider a pseudo-hyperoval $\mathcal{O}$ in $\mathbf{P G}(3 n-1, q), q=2^{h}, h>1$ and $n$ prime. Then $\mathcal{O}$ is regular if and only if all $(n-1)$-spreads $\Delta_{i}$, with $i=0,1, \cdots, q^{n}+1$, are regular.

Proof. If $\mathcal{O}$ is regular, then clearly all $(n-1)$-spreads $\Delta_{i}$, with $i=0,1, \cdots, q^{n}+1$, are regular.

Conversely, assume that the $(n-1)$-spreads $\Delta_{0}, \Delta_{1}, \cdots, \Delta_{q^{n}+1}$ are regular. Let $\mathcal{O}=\left\{\pi_{0}, \pi_{1}, \cdots, \pi_{q^{n}+1}\right\}$ and let $\hat{\mathcal{O}}=\left\{\beta_{0}, \beta_{1}, \cdots, \beta_{q^{n}+1}\right\}$ be the dual of $\mathcal{O}$, with $\beta_{i}$ being the dual of $\pi_{i}$.

Choose $\beta_{i}, i \in\left\{0,1, \cdots, q^{n}+1\right\}$, and let $\beta_{i} \cap \beta_{j}=\alpha_{i j}, j \neq i$. Then

$$
\left\{\alpha_{i 0}, \alpha_{i 1}, \cdots, \alpha_{i, i-1}, \alpha_{i, i+1}, \cdots, \alpha_{i, q^{n}+1}\right\}=\Gamma_{i}
$$

is an $(n-1)$-spread of $\beta_{i}$.

Now consider $\beta_{i}, \beta_{j}, \Gamma_{i}, \Gamma_{j}, \alpha_{i j}, j \neq i$. In $\Gamma_{j}$ we next consider a $(n-1)$-regulus $\gamma_{j}$ containing $\alpha_{i j}$. The $(n-1)$-regulus $\gamma_{j}$ is a set of maximal spaces of a Segre variety $\mathcal{S}_{1 ; n-1}$; see Section 4.5 in [4]. The $(n-1)$-regulus $\gamma_{j}$ and the $(n-1)$-spread $\Gamma_{i}$ of $\beta_{i}$ generate a regular $(n-1)$-spread $\Sigma\left(\gamma_{j}, \Gamma_{i}\right)$ of $\mathbf{P G}(3 n-1, q)$. This can be seen as follows. The elements of $\Gamma_{i}$ intersect $n$ lines $U_{1}, U_{2}, \cdots, U_{n}$ which are conjugate in $\mathbb{F}_{q^{n}}$ over $\mathbb{F}_{q}$, that is, they form an orbit of the Galois group corresponding to this extension. Let $\alpha_{i j} \cap U_{l}=\left\{u_{l}\right\}$, with $l=1,2, \cdots, n$. Now consider the transversals $T_{1}, T_{2}, \cdots, T_{n}$ of the elements of $\gamma_{j}$, with $T_{l}$ containing $u_{l}$. The $n$ planes $T_{l} U_{l}=\theta_{l}$ intersect all elements of $\gamma_{j}$ and $\Gamma_{i}$. The $(n-1)$-dimensional subspaces of $\mathbf{P G}(3 n-1, q)$ intersecting $\theta_{1}, \theta_{2}, \cdots, \theta_{n}$ are the elements of the regular $(n-1)$-spread $\Sigma\left(\gamma_{j}, \Gamma_{i}\right)$. The elements of this spread correspond to the points of a plane $\mathbf{P G}\left(2, q^{n}\right)$, with its lines corresponding to the $(2 n-1)$-dimensional spaces containing at least two (and then $q^{n}+1$ ) elements of the spread. Hence the $q+2$ elements of $\hat{\mathcal{O}}$ containing an element of $\gamma_{j}$, say $\beta_{i}=\beta_{i_{1}}, \beta_{i_{2}}, \cdots, \beta_{i_{q+1}}, \beta_{i_{q+2}}=\beta_{j}$, correspond to lines of $\mathbf{P G}\left(2, q^{n}\right)$. Dualizing, the elements $\pi_{i_{1}}, \pi_{i_{2}}, \cdots, \pi_{i_{q+2}}$ correspond to points of $\mathbf{P G}\left(2, q^{n}\right)$.

Now consider $\beta_{i_{2}}$ and $\gamma_{j}$, and repeat the argument. Then there arise $n$ planes $\theta_{l}^{\prime}$ intersecting all elements of $\gamma_{j}$ and $\Gamma_{i_{2}}$. The $(n-1)$-dimensional subspaces of $\mathbf{P G}(3 n-1, q)$ intersecting $\theta_{1}^{\prime}, \theta_{2}^{\prime}, \cdots, \theta_{n}^{\prime}$ are the elements of the regular $(n-1)$ spread $\Sigma\left(\gamma_{j}, \Gamma_{i_{2}}\right)$. The elements of this spread correspond to the points of a plane $\mathbf{P G}^{\prime}\left(2, q^{n}\right)$, and the lines of this plane correspond to the $(2 n-1)$-dimensional spaces containing $q^{n}+1$ elements of the spread. Hence $\beta_{i_{1}}, \beta_{i_{2}}, \cdots, \beta_{i_{q+2}}$ correspond to lines of $\mathbf{P G}^{\prime}\left(2, q^{n}\right)$. Dualizing, the elements $\pi_{i_{1}} . \pi_{i_{2}}, \cdots, \pi_{i_{q+2}}$ correspond to points of $\mathbf{P G}^{\prime}\left(2, q^{n}\right)$.

First, assume that $\left\{\theta_{1}, \theta_{2}, \cdots, \theta_{n}\right\} \cap\left\{\theta_{1}^{\prime}, \theta_{2}^{\prime}, \cdots, \theta_{n}^{\prime}\right\}=\emptyset$. Consider $\pi_{i_{1}}, \pi_{i_{2}}, \pi_{i_{3}}, \pi_{i_{4}}$. The planes of $\mathbf{P G}\left(3 n-1, q^{n}\right)$ intersecting these four spaces constitute a set $\mathcal{M}$ of 
maximal spaces of a Segre variety $\mathcal{S}_{2 ; n-1}[1]$. The planes $\theta_{1}, \theta_{2}, \cdots, \theta_{n}, \theta_{1}^{\prime}, \theta_{2}^{\prime}, \cdots, \theta_{n}^{\prime}$ are elements of $\mathcal{M}$. It follows that $\left(\theta_{1} \cup \theta_{2} \cup \cdots \cup \theta_{n}\right) \cap\left(\theta_{1}^{\prime} \cup \theta_{2}^{\prime} \cup \cdots \cup \theta_{n}^{\prime}\right)=\emptyset$.

Consider any $(n-1)$-dimensional subspace $\pi \in\left\{\pi_{i_{5}}, \pi_{i_{6}}, \cdots, \pi_{i_{q+2}}\right\}$ of $\mathbf{P G}(3 n-$ $1, q)$. We will show that $\pi$ is a maximal subspace of $\mathcal{S}_{2 ; n-1}$. Let $\theta_{i} \cap \pi_{j}=\left\{t_{i j}\right\}, \theta_{i}^{\prime} \cap$ $\pi_{j}=\left\{t_{i j}^{\prime}\right\}, i=1,2, \cdots, n, j=i_{1}, i_{2}, \cdots, i_{q+2}$. If $t_{i j_{1}} t_{i j_{2}} \cap t_{i j_{3}} t_{i j_{4}}=\left\{v_{i}\right\}, t_{i j_{1}}^{\prime} t_{i j_{2}}^{\prime} \cap$ $t_{i j_{3}}^{\prime} t_{i j_{4}}^{\prime}=\left\{v_{i}^{\prime}\right\}$, with $j_{1}, j_{2}, j_{3}, j_{4}$ distinct, then $v_{1}, v_{2}, \cdots, v_{n}$ are conjugate and similarly $v_{1}^{\prime}, v_{2}^{\prime}, \cdots, v_{n}^{\prime}$ are conjugate. Hence $\left\langle v_{1}, v_{2}, \cdots, v_{n}\right\rangle=\left\langle v_{1}^{\prime}, v_{2}^{\prime}, \cdots, v_{n}^{\prime}\right\rangle$ defines a $(n-1)$-dimensional space over $\mathbb{F}_{q}$ which intersects $\theta_{1}, \theta_{2}, \cdots, \theta_{n}^{\prime}$ (over $\mathbb{F}_{q^{n}}$ ). The points $t_{i j}$, with $j=i_{1}, i_{2}, \cdots, i_{q+2}$, generate a subplane of $\theta_{i}$, and the points $t_{i j}^{\prime}$, with $j=i_{1}, i_{2}, \cdots, i_{q+2}$, generate a subplane of $\theta_{i}^{\prime}$, with $i=1,2, \cdots, n$. Let $q=2^{h}$ and let $\mathbb{F}_{2^{v}}$ be the subfield of $\mathbb{F}_{q^{n}}=\mathbb{F}_{2^{h n}}$ over which these subplanes are defined; so $v \mid h n$. Then $v<h n$ as otherwise the spreads of $\mathbf{P G}(3 n-1, q)$ defined by $\theta_{1}, \theta_{2}, \cdots, \theta_{n}$ and $\theta_{1}^{\prime}, \theta_{2}^{\prime}, \cdots, \theta_{n}^{\prime}$ coincide, clearly not possible. The $(n-1)$-regulus $\gamma_{j}$ implies that the subplanes contain a line over $\mathbb{F}_{q}$, so $h \mid v$. As $n$ is prime we have $v=h$, so $2^{v}=q$. Hence the $2 n$ subplanes are defined over $\mathbb{F}_{q}$. It follows that the $q+2$ elements $\pi_{i_{1}}, \pi_{i_{2}}, \cdots, \pi_{i_{q+2}}$ are maximal subspaces of the Segre variety $\mathcal{S}_{2 ; n-1}$. Hence $\pi$ is a maximal subspace of $\mathcal{S}_{2 ; n-1}$. It follows that $\pi_{1}, \pi_{2}, \cdots, \pi_{q+2}$ are maximal subspaces of $\mathcal{S}_{2 ; n-1}$.

Now consider a $\mathbf{P G}(2, q)$ which intersects $\pi_{i_{1}}, \pi_{i_{2}}, \pi_{i_{3}}, \pi_{i_{4}}$. The $(n-1)$-dimensional spaces $\pi_{i_{1}}, \pi_{i_{2}}, \cdots, \pi_{i_{q+2}}$ are maximal spaces of $\mathcal{S}_{2 ; n-1}$ which intersect $\mathbf{P G}(2, q)$; they are maximal spaces of the Segre variety $\mathcal{S}_{2 ; n-1} \cap \mathbf{P G}(3 n-1, q)$ of $\mathbf{P G}(3 n-1, q)$.

Consider $\pi_{i_{1}}$ and also a $\mathbf{P G}(2 n-1, q)$ skew to $\pi_{i_{1}}$. If we project $\pi_{i_{2}}, \pi_{i_{3}}, \cdots, \pi_{i_{q+2}}$ from $\pi_{i_{1}}$ onto $\mathbf{P G}(2 n-1, q)$, then by the foregoing paragraph the $q+1$ projections constitute a $(n-1)$-regulus of $\mathbf{P G}(2 n-1, q)$. Similarly, if we project from $\pi_{i_{s}}, s$ any element of $\{1,2, \cdots, q+2\}$. Equivalently, if $s \in\{1,2, \cdots, q+2\}$ then the spaces $\beta_{i_{s}} \cap \beta_{i_{t}}$, with $t=1,2, \cdots, s-1, s+1, \cdots, q+2$, form a $(n-1)$-regulus of $\beta_{i_{s}}$.

Now assume that the condition $\left\{\theta_{1}, \theta_{2}, \cdots, \theta_{n}\right\} \cap\left\{\theta_{1}^{\prime}, \theta_{2}^{\prime}, \cdots, \theta_{n}^{\prime}\right\}=\emptyset$ is satisfied for any choice of $\beta_{i}, \beta_{j}, \gamma_{j}, \beta_{i_{2}}$. In such a case every $(n-1)$-regulus contained in a spread $\Gamma_{s}$ defines a Segre variety $\mathcal{S}_{2 ; n-1}$ over $\mathbb{F}_{q}$. Let us define the following design $\mathcal{D}$. Points of $\mathcal{D}$ are the elements of $\hat{\mathcal{O}}$, a block of $\mathcal{D}$ is a set of $q+2$ elements of $\hat{\mathcal{O}}$, containing at least one space of a $(n-1)$-regulus contained in some regular spread $\Gamma_{s}$, and incidence is containment. Then $\mathcal{D}$ is a $4-\left(q^{n}+2, q+2,1\right)$ design. By Kantor [5] this implies that $q=2$, a contradiction.

Consequently, we may assume that for at least one quadruple $\beta_{i}, \beta_{j}, \gamma_{j}, \beta_{i_{2}}$ we have

$$
\left\{\theta_{1}, \theta_{2}, \cdots, \theta_{n}\right\}=\left\{\theta_{1}^{\prime}, \theta_{2}^{\prime}, \cdots, \theta_{n}^{\prime}\right\} .
$$

In such a case the $q^{n}+2$ elements of $\hat{\mathcal{O}}$ correspond to lines of the plane $\mathbf{P G}\left(2, q^{n}\right)$. It follows that $\mathcal{O}$ is regular.

Theorem 6.2. Consider a pseudo-oval $\mathcal{O}$ in $\mathbf{P G}(3 n-1, q)$, with $q=2^{h}, h>1$ and $n$ prime. Then $\mathcal{O}$ is regular if and only if all $(n-1)$-spreads $\Delta_{0}, \Delta_{1}, \cdots, \Delta_{q^{n}}$ are regular.

Proof. If $\mathcal{O}$ is regular, then clearly all $(n-1)$-spreads $\Delta_{0}, \Delta_{1}, \cdots, \Delta_{q^{n}}$ are regular. Conversely, assume that the $(n-1)$-spreads $\Delta_{0}, \Delta_{1}, \cdots, \Delta_{q^{n}}$ are regular. Let $\mathcal{O}=$ $\left\{\pi_{0}, \pi_{1}, \cdots, \pi_{q^{n}}\right\}$, let $\pi_{q^{n}+1}$ be the nucleus of $\mathcal{O}$, let $\overline{\mathcal{O}}=\mathcal{O} \cup\left\{\pi_{q^{n}+1}\right\}$, let $\hat{\mathcal{O}}$ be the dual of $\mathcal{O}$, let $\hat{\overline{\mathcal{O}}}$ be the dual of $\overline{\mathcal{O}}$, and let $\beta_{i}$ be the dual of $\pi_{i}$. 
Choose $\beta_{i}, i \in\left\{0,1, \cdots, q^{n}+1\right\}$, and let $\beta_{i} \cap \beta_{j}=\alpha_{i j}, j \neq i$. Then

$$
\left\{\alpha_{i 0}, \alpha_{i 1}, \cdots, \alpha_{i, i-1}, \alpha_{i, i+1}, \cdots, \alpha_{i, q^{n}+1}\right\}=\Gamma_{i}
$$

is an $(n-1)$-spread of $\beta_{i}$.

Now consider $\beta_{i}, \beta_{j}, \Gamma_{i}, \Gamma_{j}, \alpha_{i j}$, with $j \neq i$ and $i, j \in\left\{0,1, \cdots, q^{n}\right\}$. In $\Gamma_{j}$ we next consider a $(n-1)$-regulus $\gamma_{j}$ containing $\alpha_{i j}$ and $\alpha_{j, q^{n}+1}$. The $(n-1)$-regulus $\gamma_{j}$ is a set of maximal spaces of a Segre variety $S_{1 ; n-1}$. The $(n-1)$-regulus $\gamma_{j}$ and the $(n-1)$-spread $\Gamma_{i}$ of $\beta_{i}$ generate a regular $(n-1)$-spread $\Sigma\left(\gamma_{j}, \Gamma_{i}\right)$ of $\mathbf{P G}(3 n-1, q)$. Such as in the proof of Theorem 6.1 we introduce the elements $U_{l}, u_{l}, T_{l}, \theta_{l}, l=$ $1,2, \cdots, n$, and the plane $\mathbf{P G}\left(2, q^{n}\right)$. The $q+2$ elements of $\hat{\overline{\mathcal{O}}}$ containing an element of $\gamma_{j}$, say $\beta_{i}=\beta_{i_{1}}, \beta_{i_{2}}, \cdots, \beta_{i_{q}}, \beta_{j}=\beta_{i_{q+1}}, \beta_{q^{n}+1}$, correspond to lines of $\mathbf{P G}\left(2, q^{n}\right)$. Dualizing, the elements $\pi_{i_{1}}, \pi_{i_{2}}, \cdots, \pi_{i_{q+1}}, \pi_{q^{n}+1}$ correspond to points of $\mathbf{P G}\left(2, q^{n}\right)$.

Now consider $\beta_{i_{2}}$ and $\gamma_{j}$, and repeat the argument. Then there arise $n$ planes $\theta_{l}^{\prime}$ of $\mathbf{P G}\left(3 n-1, q^{n}\right)$ intersecting all elements of $\gamma_{j}$ and $\Gamma_{i_{2}}$, and a $(n-1)$-spread $\Sigma\left(\gamma_{j}, \Gamma_{i_{2}}\right)$ of $\mathbf{P G}(3 n-1, q)$. The elements of this spread correspond to the points of a plane $\mathbf{P G}^{\prime}\left(2, q^{n}\right)$. The spaces $\beta_{i_{1}}, \beta_{i_{2}}, \cdots, \beta_{i_{q+1}}, \beta_{q^{n}+1}$ correspond to lines of $\mathbf{P G}^{\prime}\left(2, q^{n}\right)$. Dualizing, the elements $\pi_{i_{1}}, \pi_{i_{2}}, \cdots, \pi_{i_{q+1}}, \pi_{q^{n}+1}$ correspond to points of $\mathbf{P G}^{\prime}\left(2, q^{n}\right)$.

First, assume that $\left\{\theta_{1}, \theta_{2}, \cdots, \theta_{n}\right\} \cap\left\{\theta_{1}^{\prime}, \theta_{2}^{\prime}, \cdots, \theta_{n}^{\prime}\right\}=\emptyset$. Consider $\pi_{i_{1}}, \pi_{i_{2}}, \pi_{i_{3}}, \pi_{i_{4}}$. The planes of $\mathbf{P G}\left(3 n-1, q^{n}\right)$ intersecting these four spaces constitute a set $\mathcal{M}$ of maximal spaces of a Segre variety $\mathcal{S}_{2 ; n-1}$. The planes $\theta_{1}, \theta_{2}, \cdots, \theta_{n}, \theta_{1}^{\prime}, \theta_{2}^{\prime}, \cdots, \theta_{n}^{\prime}$ are elements of $\mathcal{M}$. It follows that $\left(\theta_{1} \cup \theta_{2} \cup \cdots \cup \theta_{n}\right) \cap\left(\theta_{1}^{\prime} \cup \theta_{2}^{\prime} \cup \cdots \cup \theta_{n}^{\prime}\right)=\emptyset$. Let $\pi \in\left\{\pi_{i_{5}}, \pi_{i_{6}}, \cdots, \pi_{i_{q+1}}, \pi_{q^{n}+1}\right\}$. As in the proof of Theorem 6.1 one shows that $\pi$ is a maximal subspace of $\mathcal{S}_{2 ; n-1}$. It follows that $\pi_{i_{1}}, \pi_{i_{2}}, \cdots, \pi_{i_{q+1}}, \pi_{q^{n}+1}$ are maximal subspaces of $\mathcal{S}_{2 ; n-1}$.

Next consider a PG $(2, q)$ which intersects $\pi_{i_{1}}, \pi_{i_{2}}, \pi_{i_{3}}, \pi_{i_{4}}$. The $(n-1)$-dimensional spaces $\pi_{i_{1}}, \pi_{i_{2}}, \cdots, \pi_{i_{q+1}}, \pi_{q^{n}+1}$ are maximal spaces of $\mathcal{S}_{2 ; n-1}$ which intersect the plane $\mathbf{P G}(2, q)$; they are maximal spaces of the Segre variety $\mathcal{S}_{2 ; n-1} \cap \mathbf{P G}(3 n-$ $1, q)$ of $\mathbf{P G}(3 n-1, q)$. As in the proof of Theorem 6.1 it follows that the spaces $\beta_{q^{n}+1} \cap \beta_{i_{t}}$, with $t=1,2, \cdots, q+1$, form a $(n-1)$-regulus of $\beta_{q^{n}+1}$.

Now assume that the condition $\left\{\theta_{1}, \theta_{2}, \cdots, \theta_{n}\right\} \cap\left\{\theta_{1}^{\prime}, \theta_{2}^{\prime}, \cdots, \theta_{n}^{\prime}\right\}=\emptyset$ is satisfied for any choice of $\beta_{i}, \beta_{j}, \gamma_{j}, \beta_{i_{2}}, j \neq i$ and $i, j \in\left\{0,1, \cdots, q^{n}\right\}$. Let $\alpha_{1}, \alpha_{2}, \alpha_{3}$ be distinct elements of $\Gamma_{q^{n}+1}$. Then $\beta_{i}, \beta_{j}, \gamma_{j}, \beta_{i_{2}}$ can be chosen in such a way that $\alpha_{1} \in \beta_{i}, \alpha_{2} \in \beta_{j}, \alpha_{2} \in \gamma_{j}, \beta_{i_{2}} \cap \beta_{j} \in \gamma_{j}$ with $\alpha_{3} \in \beta_{i_{2}}$. Hence the $(n-1)$-regulus in $\beta_{q^{n}+1}$ defined by $\alpha_{1}, \alpha_{2}, \alpha_{3}$ is subset of $\Gamma_{q^{n}+1}$. From [4], Theorem 4.123, now follows that the $(n-1)$-spread $\Gamma_{q^{n}+1}$ of $\beta_{q^{n}+1}$ is regular. By Theorem 6.1 the pseudo-hyperoval $\overline{\mathcal{O}}$ is regular, and so $\mathcal{O}$ is regular. But in such a case the condition $\left\{\theta_{1}, \theta_{2}, \cdots, \theta_{n}\right\} \cap\left\{\theta_{1}^{\prime}, \theta_{2}^{\prime}, \cdots, \theta_{n}^{\prime}\right\}=\emptyset$ is never satisfied, a contradiction.

Consequently, we may assume that for at least one quadruple $\beta_{i}, \beta_{j}, \gamma_{j}, \beta_{i_{2}}$ we have $\left\{\theta_{1}, \theta_{2}, \cdots, \theta_{n}\right\}=\left\{\theta_{1}^{\prime}, \theta_{2}^{\prime}, \cdots, \theta_{n}^{\prime}\right\}$. In such a case the $q^{n}+2$ elements of $\hat{\overline{\mathcal{O}}}$ correspond to lines of the plane $\mathbf{P G}\left(2, q^{n}\right)$. It follows that $\overline{\mathcal{O}}$, and hence also $\mathcal{O}$, is regular.

Theorem 6.3. Consider a pseudo-hyperoval $\mathcal{O}$ in $\mathbf{P G}(3 n-1, q), q=2^{h}, h>1$ and $n$ prime. Then $\mathcal{O}$ is regular if and only if at least $q^{n}-1$ elements of $\left\{\Delta_{0}, \Delta_{1}, \cdots, \Delta_{q^{n}+1}\right\}$ are regular. 
Proof. If $\mathcal{O}$ is regular, then clearly all $(n-1)$-spreads $\Delta_{i}$, with $i=0,1, \cdots, q^{n}+1$, are regular.

Conversely, assume that $\rho$, with $\rho \geq q^{n}-1$, elements of $\left\{\Delta_{0}, \Delta_{1}, \cdots, \Delta_{q^{n}+1}\right\}$ are regular.

If $\rho=q^{n}+2$, then $\mathcal{O}$ is regular by Theorem 6.1; if $\rho=q^{n}+1$, then $\mathcal{O}$ is regular by Theorem 6.2.

Now assume that $\rho=q^{n}$ and that $\Delta_{2}, \Delta_{3}, \cdots, \Delta_{q^{n}+1}$ are regular. We have to prove that $\Delta_{0}$ is regular. We use the arguments in the proof of Theorem 6.2. If one of the elements $\alpha_{1}, \alpha_{2}, \alpha_{3}$, say $\alpha_{1}$, in the proof of Theorem 6.2 is $\beta_{0} \cap \beta_{1}$, then let $\gamma_{j}$ contain $\beta_{j} \cap \beta_{i}, \beta_{j} \cap \beta_{0}, \beta_{j} \cap \beta_{1}$ and let $\beta_{i_{2}} \neq \beta_{1}$, with $i, j \in\left\{2,3, \cdots, q^{n}+1\right\}$. Now see the proof of the preceding theorem.

Finally, assume that $\rho=q^{n}-1$ and that $\Delta_{3}, \Delta_{4}, \cdots, \Delta_{q^{n}+1}$ are regular. We have to prove that $\Delta_{0}$ is regular. We use the arguments in the proof of Theorem 6.2. If exactly one of the elements $\alpha_{1}, \alpha_{2}, \alpha_{3}$, say $\alpha_{1}$, in the proof of Theorem 6.2 is $\beta_{0} \cap \beta_{1}$ or $\beta_{0} \cap \beta_{2}$, then proceed as in the preceding paragraph with $\beta_{i_{2}} \neq \beta_{1}, \beta_{2}$. Now assume that two of the elements $\alpha_{1}, \alpha_{2}, \alpha_{3}$, say $\alpha_{1}$ and $\alpha_{2}$, are $\beta_{0} \cap \beta_{1}$ and $\beta_{0} \cap \beta_{2}$. Now consider all $(n-1)$-reguli in $\Delta_{0}$ containing $\alpha_{1}$ and $\alpha_{3}$, and assume, by way of contradiction, that no one of these $(n-1)$-reguli contains $\alpha_{2}$. The number of these $(n-1)$-reguli is $\frac{q^{n}-2}{q-1}$, and so $q=2$, a contradiction. It follows that the $(n-1)$-regulus in $\beta_{0}$ defined by $\alpha_{1}, \alpha_{2}, \alpha_{3}$ is contained in $\Delta_{0}$. Now we proceed as in the proof of Theorem 6.2.

\section{FinAL REMARKS}

\subsection{The cases $q=2$ and $n$ not prime}

For $q=2$ or $n$ not prime other arguments have to be developed.

\subsection{Improvement of Theorem 6.3}

Let $\mathcal{D}=(P, B, \in)$ be an incidence structure satisfying the following conditions.

(i) $|P|=q^{n}+1, q$ even, $q \neq 2$;

(ii) the elements of $B$ are subsets of size $q+1$ of $P$ and every three distinct elements of $P$ are contained in at most one element of $B$;

(iii) $\mathrm{Q}$ is a subset of size $\delta$ of $P$ such that any triple of elements in $P$ with at most one element in $Q$, is contained in exactly one element of $B$.

Assumption : Any such $\mathcal{D}$ is a $3-\left(q^{n}+1, q+1,1\right)$ design whenever $\delta \leq \delta_{0}$ with $\delta_{0} \leq q-2$.

Theorem 7.1. Consider a pseudo-hyperoval $\mathcal{O}$ in $\mathbf{P G}(3 n-1, q), q=2^{h}, h>1$ and $n$ prime. Then $\mathcal{O}$ is regular if and only if at least $q^{n}+1-\delta_{0}$ elements of $\left\{\Delta_{0}, \Delta_{1}, \cdots, \Delta_{q^{n}+1}\right\}$ are regular.

Proof. Similar to the proof of Theorem 6.3.

\subsection{Acknowledgement}

We thank S. Rottey and G. Van de Voorde for several helpful discussions. 


\section{REFERENCES}

[1] W. Burau. Mehrdimensionale Projective und Höhere Geometrie, VEB Deutscher Verlag der Wissenschaften, Berlin, 1961, 436 pp.

[2] L. R. A. Casse, J. A. Thas and P. R. Wild. $\left(q^{n}+1\right)$-sets of $\mathbf{P G}(3 n-1, q)$, generalized quadrangles and Laguerre planes, Simon Stevin 59 (1985), 21 - 42.

[3] J. W. P. Hirschfeld. Projective Geometries over Finite Fields, Oxford University Press, Oxford, second edition, 1998, xiv +555 pp.

[4] J. W. P. Hirschfeld and J. A. Thas. General Galois Geometries, Springer-Verlag, London, second edition, 2016, xvi + 409 pp.

[5] W. M. Kantor. Dimension and embedding theorems for geometric lattices, J. Combin. Theory Ser. A 17 (1974) 173 - 195.

[6] S. E. Payne and J. A. Thas. Finite Generalized Quadrangles, European Mathematical Society, Zurich, second edition, 2009, xii +287 pp.

[7] T. Penttila and G. Van de Voorde. Extending pseudo-arcs in odd characteristic, Finite Fields Appl. 22 (2013) $101-113$.

[8] S. Rottey and G. Van de Voorde. Pseudo-ovals in even characteristic and ovoidal Laguerre planes, J. Combin. Theory Ser. A 129 (2015) 105 - 121.

[9] B. Segre. Sulle ovali nei piani lineari finiti, Atti Accad Naz. Lincei, Rend. 17 (1954) 1 - 2.

[10] J. A. Thas. The $m$-dimensional projective space $S_{m}\left(M_{n}(G F(q))\right)$ over the total matrix algebra $M_{n}(G F(q))$ of the $(n \times n)$-matrices with elements in the Galois field $G F(q)$, Rend. Mat. 4 (1971) $459-532$.

[11] J. A. Thas. Generalized ovals in PG(3n-1,q), with $q$ odd, Pure Appl. Math. Q. 7 (2011) 1007 1035 (Special Issue: In honor of Jacques Tits).

[12] J. A. Thas, K. Thas and H. Van Maldeghem. Translation Generalized Quadrangles, Ser. Pure Math., vol. 6, World Sci. Publ., Hackensack, 2006, xxx + 345 pp.

Ghent University, Department of Mathematics, KriJgslaAn 281, S22, B-9000 Ghent, Belgium

Email address: jat@cage.ugent.be 